\title{
SURFACE EQUIVALENCE PRINCIPLE AND SURFACE INTEGRAL EQUATION (SIE) REVISITED FOR BIOELECTROMAGNETICS APPLICATION
}

\author{
M. CVETKOVIĆ \& D. POLJAK \\ University of Split, Faculty of Electrical Engineering, Mechanical Engineering and Naval Architecture, Croatia.
}

\begin{abstract}
The paper revisits the use of a surface equivalence theorem in deriving the surface integral equation (SIE) based formulation for a homogeneous bio-electromagnetics problem. The vector analog of Green's 2nd identity is used to obtain the expression for the electric field representing the mathematical foundation of the equivalence theorem. The particular emphasis is put on the treatment of boundary integral when the observation and source points, respectively, coincide. The boundary conditions at infinity are taken into account via the Sommerfeld radiation conditions. The derived coupled SIE set can be used in problems involving biological body exposed to electromagnetic field radiation.

Keywords: Computational electromagnetics, Equivalence principle, Human exposure to electromagnetic fields, Surface integral equation
\end{abstract}

\section{INTRODUCTION}

The human exposure to electromagnetic (EM) fields generated by different sources such as power and telecommunication installations has raised a number of questions regarding potentially adverse health effects. In addition to unwanted exposure to artificially generated EM fields, some biomedical applications of EM fields in medical diagnostics and for therapy purposes, has recently become of particular importance. In order to address the concern regarding the potentially harmful effects, but also to aid in the design of a more efficient medical treatments using EM radiation, the knowledge of the accurate distribution of the electromagnetic fields inside the biological tissues is required. As it is rather difficult, if possible at all, to accurately measure the induced fields within the living biological body, the exposure assessment is carried out using a sophisticated computational bioelectromagnetics models [1-3].

From the early days, bioelectromagnetics models based on differential equation approach has become the de facto standard, although the integral equation approach, using the Green integral representation, is suitable for the exact treatment of open boundary problems such as the human head or the body exposed to incident EM field $[4,5]$. Regardless of the fact that the numerical methods based on the solution of integral equations in computational electromagnetics (CEM) were developed during the sixties [6, 7], only recently has this approach seen a revival in bioelectromagnetics community $[2,4,5,8,9]$.

One interesting approach to formulate bioelectromagnetics problem is similar to a classical scattering problem [4]. If one considers the biological body of an arbitrary shape, placed in the field of an incident EM wave, an EM model based on the integral equation can be posed by using the equivalence theorem and the appropriate boundary conditions. In the case of scattering from a homogeneous dielectric body, the surface integral equation (SIE), based on the application of surface equivalence theorem is used, while in the non-homogeneous case, the appropriate volume integral equation (VIE) is derived using the volume equivalence principle. 
This paper aims to revisit the applications of the surface equivalence theorem and the derivation of electric field integral equation (EFIE) formulation applicable to bioelectromagnetics problem. The paper is organized as follows: The first part deals with vector analog of the Green's theorem used to derive the expression for the so called Stratton-Chu expression. This is followed by a treatment of the limiting case when observation and source points, respectively, coincide. The boundary conditions at infinity are taken into account via the Sommerfeld radiation conditions. Furthermore, the application of the equivalence principle is demonstrated to result in the coupled integral equations set. The final part presents some illustrative numerical results obtained by using SIE formulation related to bioelectromagnetics application.

\section{ON THE USE OF GREEN'S SECOND IDENTITY}

Let us consider the problem depicted in Fig. 1 . The lossy material body $V_{2}$ of complex parameters $\left(\varepsilon_{2}, \mu_{2}\right)$, bounded by surface $S$, is placed in an infinite region $V_{1}$, with complex parameters $\left(\varepsilon_{1}, \mu_{1}\right)$, bounded with $S_{1}$, where $S_{1}=S \cup S_{\varepsilon} \cup S_{\infty}$, with electric and magnetic source currents $\left(\vec{J}_{1}, \vec{M}_{1}\right) . S_{\infty}$ and $S_{\varepsilon}$ represent the boundary at the infinity, and of infinitesimal space $V_{\varepsilon}$ required when $\vec{r} \rightarrow \vec{r}^{\prime}$, respectively. $\vec{r}$ and $\vec{r}^{\prime}$ represent the observation and the source point, respectively. Unit vector $\hat{n}$ is directed from $V_{2}$ to $V_{1}$, while unit vectors $\hat{n}_{1}$ and $\hat{n}_{2}$, respectively, are outward directed for each region.

If no material body $V_{2}$ is present, the field due to the currents induced by radiating source at an arbitrary point in $V_{1}$ is relatively easy to determine. However, due to the presence of a material body $V_{2}$, the field is perturbed, leading to a significantly complex problem, since it is necessary to satisfy the boundary conditions at the interface between two regions.

One way of solving such a problem is to link the field inside $V_{1}$ with sources within that region, and the field on the boundary surface of $S_{1}$. To facilitate this connection, the vector analog of Green's theorem or Green's 2nd identity is used:

$$
\int_{V}(\vec{Q} \cdot \nabla \times \nabla \times \vec{P}-\vec{P} \cdot \nabla \times \nabla \times \vec{Q}) d V=\int_{S}(\vec{P} \cdot \nabla \times \nabla \times \vec{Q}-\vec{Q} \cdot \nabla \times \nabla \times \vec{P}) \cdot n d S
$$

where $\vec{P}$ and $\vec{Q}$ are vector functions with continuous first and second derivatives within $V_{1}$, satisfying Helmholtz equation, selected as:

$$
\vec{P}=\hat{a} G\left(\vec{r}, \vec{r}^{\prime}\right) ; \quad \vec{Q}=\vec{E}(\vec{r})
$$

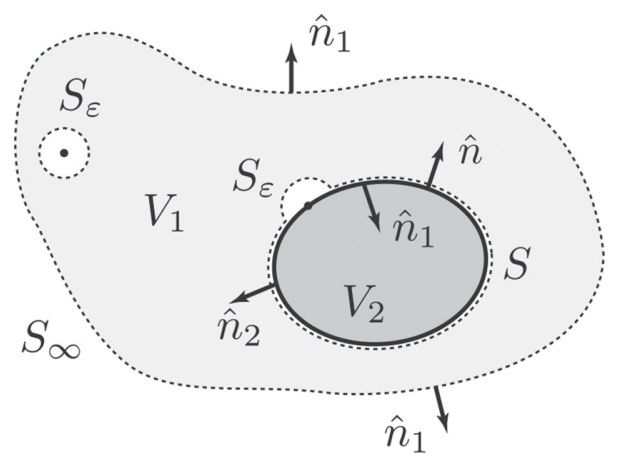

Figure 1: Problem description with denoted regions and boundaries. 
where $\vec{E}$ is the electric fields, $\hat{a}$ is the consatnty unit vector, while $G\left(\vec{r}, \vec{r}^{t}\right)$ denotes the free space scalar Green's function

$$
G\left(\vec{r}, \vec{r}^{\prime}\right)=\frac{e^{-j k R}}{4 \pi R} ; \quad R=\left|\vec{r}-\vec{r}^{\prime}\right|
$$

In (3), $\mathrm{R}$ is the distance from the observation point $\vec{r}$ to the source point $\vec{r}^{\prime}$, and $k=\omega \sqrt{\mu \omega}$ is a wave number in free space.

2.1 Left hand side of the identity

Substituting (2) in (1), after some manipulation, the first term on the left side of (1) can be written as:

$$
\nabla \times \nabla \times \vec{P}=\nabla \times \nabla \times \hat{a} G=\nabla(\hat{a} \cdot \nabla G)+k^{2} G
$$

where $\mu$ and $\varepsilon$ are permitivity and permeability, respectively, and $\omega=2 \pi f$ is operating frequency.

Multiplying (4) with $\vec{E}$ and integrating over volume $V_{1}$, leads to the following expression

$$
I=\int_{V_{1}} \vec{E} \cdot\left[\nabla(a \cdot \nabla G)+a k^{2} G\right] d V
$$

which, after performing some mathematical manipulations results in

$$
I=\int_{S_{1}} \vec{E} \cdot(a \cdot \nabla G) n d S-\int_{V_{1}}(a \cdot \nabla G) \frac{\rho}{\varepsilon} d V+\int_{V_{1}} \vec{E} \cdot a k^{2} G d V
$$

where $\rho$ represents the electric charge density.

After some rearranging, the constant vector is moved outside the integrals, leading to the following expression

$$
I=\hat{a} \cdot \int_{S_{1}} \nabla G(\hat{n} \cdot \vec{E}) d S+\hat{a} \cdot \int_{V_{1}}\left(k^{2} G \vec{E}-\frac{\rho}{\varepsilon} \nabla G\right) d V
$$

Repeating the same procedure, the 2 nd term on the left side of (1) can be written as:

$$
\vec{P} \cdot \nabla \times \nabla \times \vec{Q}=\hat{a} \cdot G\left[k^{2} \vec{E}-j \omega \mu \vec{J}-\nabla \times \vec{M}\right]
$$

where the following substitution has been used:

$$
\nabla \times \nabla \times \vec{E}=k^{2} \vec{E}-j \omega \mu \vec{J}-\nabla \times \vec{M}
$$

Integrating (8) over volume $V_{1}$, after some rearranging, the following expression is obtained:

$$
I I=\hat{a} \cdot \int_{V_{1}} G\left(k^{2} \vec{E}-j \omega \mu \vec{J}\right) d V-\hat{a} \cdot \int_{V_{1}} G(\nabla \times \vec{M}) d V
$$

Partial integration of the last term in (10), followed by application of vector form of Stokes theorem, leads to:

$$
I I=\hat{a} \cdot \int_{V_{1}} G\left(k^{2} \vec{E}-j \omega \mu \vec{J}\right) d V-\hat{a} \cdot \int_{S_{1}} \hat{n} \times(G \vec{M}) d S+\hat{a} \cdot \int_{V_{1}} \nabla G \times \vec{M} d V
$$


Finally, subtracting (11) from (7), left side of Green's second identity (1) can be written as follows:

$$
\begin{aligned}
\text { L.H.S. } \equiv & a \cdot \int_{S_{1}} \nabla G(n \cdot \vec{E}) d S+a \cdot \int_{V_{1}}\left(k^{2} G \vec{E}-\frac{\rho}{\varepsilon} \nabla G\right) d V \\
& -a \cdot \int_{V_{1}} G\left(k^{2} \vec{E}-j \omega \mu \vec{J}\right) d V-\hat{a} \cdot \int_{S_{1}} \hat{n} \times(G \vec{M}) d S \\
& +\hat{a} \cdot \int_{V_{1}} \nabla G \times \vec{M} d V
\end{aligned}
$$

which, after some rearrangement, results in:

$$
\int_{V_{1}}\left(j \omega \mu \vec{J} G-\frac{\rho}{\varepsilon} \nabla G-\nabla G \times \vec{M}\right) d V+\int_{S_{1}}[\nabla G(n \cdot \vec{E})+n \times(G \vec{M})] d S
$$

2.2 Right hand side of the identity

Treatment of the right hand side of (1) is practically identical. The first term can be written as:

$$
\vec{P} \times \nabla \times \vec{Q}=\hat{a} G \times \nabla \times \vec{E}
$$

Where the use of $\nabla \times \vec{E}=-j \omega \mu \vec{H}-\vec{M}$ will lead to:

$$
(\vec{P} \times \nabla \times \vec{Q}) \cdot \hat{n}=\hat{a} \cdot[\hat{n} \times(j \omega \mu G \vec{H}+G \vec{M})]
$$

Repeating the same procedure, the second term from the right hand side of (1) can be written as

$$
(\vec{Q} \times \nabla \times \vec{P}) \cdot \hat{n}=[\vec{E} \times \nabla \times(\hat{a} G)] \cdot \hat{n}=\hat{a} \cdot(\hat{n} \cdot \vec{E}) \times \nabla G
$$

Integrating (15) and (16), respectively, over the surface $S_{1}$, followed by a subtraction, the following expression is obtained:

$$
\begin{aligned}
\text { R.H.S. } & \equiv \int_{S_{1}} \hat{a} \cdot[\hat{n} \times(j \omega \mu G \vec{H}+G \vec{M})] d S-\int_{S_{1}} \hat{a} \cdot(\hat{n} \cdot \vec{E}) \times \nabla G d S= \\
& =\hat{a} \cdot[-(\hat{n} \times \vec{E}) \times \nabla G+j \omega \mu G(\hat{n} \times H)+\hat{n} \times(G \vec{M})] d S
\end{aligned}
$$

Finally, by equating (13) and (17), after some additional mathematical manipulations, results in the following formula:

$$
\begin{aligned}
& \int_{V_{1}}\left[j \omega \mu \vec{J} G+\vec{M} \times \nabla^{\prime} G-\frac{\rho}{\varepsilon} \nabla^{\prime} G\right] d V^{\prime}= \\
& =\int_{S_{1}}\left[j \omega \mu G(n \times H)-(n \times \vec{E}) \times \nabla^{\prime} G-(n \cdot \vec{E}) \nabla^{\prime} G\right] d S^{\prime}
\end{aligned}
$$

In (18), additional substitution between the source and the observation points, has been carried out. 
The expression (18) is in a suitable form, as the left side contains all the sources $(\vec{J}, \vec{M}, \rho)$ within volume $V_{1}$, while the right side includes electric and magnetic field, respectively, on the boundary $S_{1}=S \cup S_{\varepsilon} \cup S \infty$ of the domain. Next, the behavior of the surface integral needs to be considered separately for each part of the boundary.

\section{REGION AROUND SINGULARITY}

In the limiting case when $\vec{r} \rightarrow \vec{r}^{\prime}$, it is necessary to determine surface integral of (18) for two special cases: when observation point is inside $V_{1}$, and when on the boundary surface $S_{1}$, respectively.

When former is the case, a small spherical region $S_{\varepsilon}$, radius of $\varepsilon$, is excluded around the observation point, as shown on Fig. 2a).

Inserting (3) in (18), the first integral on the right side of (18) vanishes, when $R \rightarrow 0$ :

$$
\lim _{R \rightarrow 0} \int_{S_{\varepsilon}} j \omega \mu \frac{e^{-j k R}}{4 \pi R}(n \times \vec{H}) \varepsilon^{2} \sin \theta d \theta d \varphi=0
$$

Leaving

$$
\lim _{R \rightarrow 0} \int_{S_{\varepsilon}}\left[-(n \times \vec{E}) \times \nabla^{\prime} G-(n \cdot \vec{E}) \nabla^{\prime} G\right] d S
$$

where the Green's function gradient is inserted in the following form

$$
\nabla G\left(\vec{r}, \vec{r}^{\prime}\right)=-(1+j k R) \frac{G\left(\vec{r}, \vec{r}^{\prime}\right)}{4 \pi R^{2}} \vec{R}
$$

Leading to:

$$
\begin{aligned}
& \lim _{R \rightarrow 0} \int_{S_{\varepsilon}}[\vec{E}(n \cdot n)-(n \cdot \vec{E}) n+(n \cdot \vec{E}) n](1+j k R) \frac{e^{-j k R}}{4 \pi R^{2}}= \\
& \lim _{R \rightarrow 0} \int_{S_{\varepsilon}}[\vec{E}](1+j k R) \frac{e^{-j k R}}{4 \pi R^{2}} d S=\frac{\vec{E}}{4 \pi} \int_{\theta=0}^{2 \pi} \int_{\varphi=0}^{\pi} \sin \theta d \theta d \varphi=\vec{E}(\vec{r})
\end{aligned}
$$

In the limiting case when $\vec{r} \rightarrow \vec{r}^{\prime}$, and the observation point is on the boundary surface $S$, the hemispherical region around the observation point needs to be excluded as shown in Fig. 2b, resulting in the following value for the above integral: $\frac{1}{2} \vec{E}(\vec{r})$.

\section{BOUNDARY CONDITIONS AT INFINITY}

The boundary conditions on the surface $S_{\infty}$, where R $\rightarrow \infty$, represents the so called Sommerfeld radiating condition. The surface integral of (18) vanishes on this surface, as easily showed if

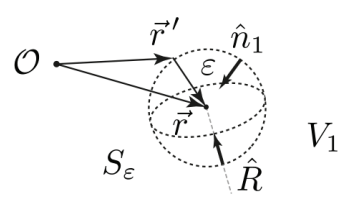

a)

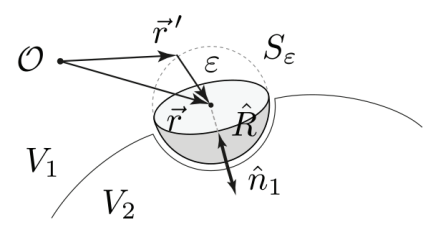

b)

Figure 2: Special case when observation point is: a) in $V_{1}$, b) on $S_{1}$. 
the surface of infinite radius $R$ is selected, with center placed at the source point $\vec{r}^{\prime}$, and radius vector $\hat{R}$ pointing outwards from $V_{1}$ :

$$
\begin{aligned}
& \lim _{R \rightarrow \infty} \int_{S_{\infty}}\{j \omega \mu G(R \times \vec{H})+\left(\frac{1}{R}+j k\right) \underbrace{[(R \times \vec{E}) \times R+(R \cdot \vec{E}) R]}_{\vec{E}} \frac{e^{-j k R}}{4 \pi R}\} R^{2} \sin \theta d \theta d \varphi= \\
& =\lim _{R \rightarrow \infty} \int_{S_{\infty}}\left[j k R(\eta R \times \vec{H}+\vec{E}) \frac{e^{-j k R}}{4 \pi}\right] \sin \theta d \theta d \varphi=0
\end{aligned}
$$

Where $\eta=\sqrt{\mu / \varepsilon}$ is the wave impedance of free space.

\section{STRATTON-CHU EXPRESSION}

Inserting (22) in (18), while replacing the surface integral designation $\left(S_{1}\right.$ changing to $S$ ), yields the integral relation for the electric field in $V_{1}$ :

$$
\begin{aligned}
\vec{E}(\vec{r}) & =\iiint_{V_{1}}\left[j \omega \mu \vec{J} G+\vec{M} \times \nabla^{\prime} G-\frac{\rho}{\varepsilon} \nabla^{\prime} G\right] d V^{\prime}+ \\
& +\iint_{S}\left[-j \omega \mu G(n \times H)+(n \times \vec{E}) \times \nabla^{\prime} G+(n \cdot \vec{E}) \nabla^{\prime} G\right] d S^{\prime}
\end{aligned}
$$

The integral expression (24) is the so called Stratton-Chu [10] solution for the electric field. The first term from (24) represents the electric field due to the sources placed inside the region $V_{1}$, while the second one is the integral representation of the sources placed outside this region expressed in terms of the field over the boundary surface $S$.

Thus, (24) enables one to consider the contribution of the sources placed outside the region of interest via their field components or the equivalent sources on the boundary surface, and represents the mathematical form of the equivalence principle [7].

\section{ON THE USE OF THE EQUIVALENCE PRINCIPLE}

Introducing the equivalent electric and magnetic current densities $\vec{J}_{S}$ and $\vec{M}_{S}$, respectively, on the surface $S$

$$
\vec{J}_{S}=\hat{n} \times \vec{H}_{1}=-\hat{n}_{1} \times \vec{H}_{1} ; \quad \vec{M}_{S}=-\hat{n} \times \vec{E}_{1}=\hat{n}_{1} \times \vec{E}_{1}
$$

the electric field in region $V_{1}$, can be expressed the following way:

$$
\begin{aligned}
& \vec{E}_{1}(\vec{r})-=\int_{V_{1}}\left[j \omega \mu_{1} \vec{J}_{1} G_{1}+\vec{M}_{1} \times \nabla^{\prime} G_{1}-\frac{\rho_{1}}{\varepsilon_{1}} \nabla^{\prime} G_{1}\right] d V^{\prime}+ \\
& \int_{S}\left[-j \omega \mu_{1} \vec{J}_{S} G_{1}+\vec{M}_{S} \times \nabla^{\prime} G_{1}-\frac{\rho_{1}}{\varepsilon_{1}} \nabla^{\prime} G_{1}\right] d S^{\prime}
\end{aligned}
$$

The last term of (26) featured the use of a continuity equation and the following substitution:

$$
\hat{n}_{1} \cdot \vec{E}_{1}=\frac{j}{\omega \varepsilon_{1}} \nabla \cdot\left(\hat{n}_{1} \times \vec{H}_{1}\right)
$$


According to (26), the electric field in $V_{1}$ is due to the sources inside this volume $\left(\vec{J}_{1}, \vec{M}_{1}\right)$, as well as due to the equivalent surface currents $\left(\vec{J}_{S}, \vec{M}_{S}\right)$, introduced to the surface $S$. Since the sources outside $V_{1}$ are omitted in (27), the entire space is homogenized, facilitating the use of a free space Green's function $G_{1}$.

The left hand side of (26) results from integral (18) evaluated over the infinitesimal surface $S_{\varepsilon}$, while it is equal to zero when (18) is evaluated at $S_{\infty}$. When the observation point is on the boundary $S$, the expression needs to be modified, resulting in:

$$
\begin{aligned}
\frac{1}{2} \vec{E}_{1}(\vec{r}) & =\int_{V_{1}}\left[j \omega \mu_{1} \vec{J}_{1} G_{1}+\vec{M}_{1} \times \nabla^{\prime} G_{1}-\frac{\rho_{1}}{\varepsilon_{1}} \nabla^{\prime} G_{1}\right] d V^{\prime}+ \\
& +\int_{S}\left[-j \omega \mu_{1} \vec{J}_{S} G_{1}-\vec{M}_{S} \times \nabla^{\prime} G_{1}+\frac{\rho S}{\varepsilon_{1}} \nabla^{\prime} \frac{\rho S}{\varepsilon_{1}} \nabla^{\prime} G_{1}\right] d S^{\prime}
\end{aligned}
$$

Finally, when the observation point is outside $V_{1}$, i.e. inside $V_{2}$, it can be readily shown that the left side of (26) vanishes [11]. Namely, when the source point and the observation point are in $V_{1}$ and $V_{2}$, respectively, it is not required to exclude the infinitesimal region around singularity, leading to:

$$
\begin{aligned}
& \int_{V_{1}}\left[j \omega \mu_{1} \vec{J}_{1} G_{1}+\vec{M}_{1} \times \nabla^{\prime} G_{1}-\frac{\rho_{1}}{\varepsilon_{1}} \nabla^{\prime} G_{1}\right] d V^{\prime}= \\
& =-\int_{S}\left[-j \omega \mu_{1} \vec{J}_{S} G_{1}-\vec{M}_{S} \times \nabla^{\prime} G_{1}+\frac{\rho_{S}}{\varepsilon_{1}} \nabla^{\prime} G_{1}\right] d S^{\prime}
\end{aligned}
$$

Subsequently inserting (29) in (26), it follows $\vec{E}_{1}(\vec{r})=0$.

On the other and, electric field in $V_{2}$ can be expressed as:

$$
\begin{aligned}
\vec{E}_{2}(\vec{r}) & =\int_{V_{2}}\left[j \omega \mu_{2} \vec{J}_{2} G_{2}+\vec{M}_{2} \times \nabla^{\prime} G_{2}-\frac{\rho_{2}}{\varepsilon_{2}} \nabla^{\prime} G_{2}\right] d V^{\prime}+ \\
& +\int_{S}\left[-j \omega \mu_{2} \vec{J}_{2} G_{2}-\vec{M}_{S} \times \nabla^{\prime} G_{2}+\frac{\rho S}{\varepsilon_{2}} \nabla^{\prime} G_{2}\right] d S^{\prime}
\end{aligned}
$$

where equivalent electric and magnetic currents are introduced on the surface $S$

$$
\vec{J}_{S}=\hat{n} \times \vec{H}_{2}=\hat{n}_{2} \times \vec{H}_{2} ; \quad \vec{M}_{S}=-\hat{n} \times \vec{E}_{2}=-\hat{n}_{2} \times \vec{E}_{2}
$$

and using $\hat{n}_{1}=-\hat{n}_{2}$.

Again, (30) states that electric field in $V_{2}$ is due to sources inside this region $\left(\vec{J}_{2}, \vec{M}_{2}\right)$, as well as some external sources taken into account via equivalent surface currents. Moreover, the whole region is homogenized again.

Similar to region 1 , it is easily shown that left hand side of (30) vanishes when observation point is outside region 2 [11], while the observation point is on surface $S$, it follows:

$$
\begin{aligned}
\frac{1}{2} \vec{E}_{2}(\vec{r})- & =\int_{V_{2}}\left[j \omega \mu_{2} \vec{J}_{2} G_{2}+\vec{M}_{2} \times \nabla^{\prime} G_{2}-\frac{\rho_{2}}{\varepsilon_{2}} \nabla^{\prime} G_{2}\right] d V^{\prime}+ \\
& +\int_{S}\left[-j \omega \mu_{2} \vec{J}_{S} G_{2}-\vec{M}_{S} \times \nabla^{\prime} G_{2}+\frac{\rho S}{\varepsilon_{2}} \nabla^{\prime} G_{2}\right] d S^{\prime}
\end{aligned}
$$


The boundary conditions for the electric field on the surface $S$ require the tangential components of vector $\vec{E}$ to be continuous. One can assume there are no field sources inside region 2 , while sources inside region 1 (volume integral over $V_{1}$ ) can be taken into account via incident field vector $\vec{E}^{i n c}$. The electric field at point $\vec{r}$, on the boundary surface $S$, from the viewpoint of region 1 and expression (28) becomes

$$
\frac{1}{2} \vec{E}_{1}(\vec{r})=\vec{E}^{i n c}+\int_{S}\left[-j \omega \mu_{1} \vec{J}_{S} G_{1}-\vec{M}_{S} \times \nabla^{\prime} G_{1}+\frac{\rho S}{\varepsilon_{1}} \nabla^{\prime} G_{1}\right] d S^{\prime}
$$

The electric field at the same point $\vec{r}$, on the boundary surface $S$, from the viewpoint of region 2 and expression (30) is:

$$
\frac{1}{2} \vec{E}_{2}(\vec{r})=\int_{S}\left[-j \omega \mu_{2} \vec{J}_{S} G_{2}-\vec{M}_{S} \times \nabla^{\prime} G_{2}+\frac{\rho S}{\varepsilon_{2}} \nabla^{\prime} G_{2}\right] d S^{\prime}
$$

Equating the left sides of (33) and (34), i.e. satisfying the boundary conditions at surface S, $\hat{n} \times \vec{E}_{1}=\hat{n} \times \vec{E}_{2}$, the right hand sides are

$$
\begin{aligned}
& -\hat{n} \times \int_{S}\left[-j \omega \mu_{1} \vec{J}_{S} G_{1}-\vec{M}_{S} \times \nabla^{\prime} G_{1}+\frac{\rho S}{\varepsilon_{1}} \nabla^{\prime} G_{1}\right] d S^{\prime}=\hat{n} \times \vec{E}^{i n c} \\
& -\hat{n} \times \int_{S}\left[-j \omega \mu_{2} \vec{J}_{S} G_{2}-\vec{M}_{S} \times \nabla^{\prime} G_{2}+\frac{\rho S}{\varepsilon_{2}} \nabla^{\prime} G_{2}\right] d S^{\prime}=0
\end{aligned}
$$

Furthermore, substituting the continuity equation into (35), yields the coupled set of SIEs, representing the EFIE formulation, which can be solved by a Moment Method (MoM) scheme, as reported in [4]:

$$
\begin{aligned}
\vec{E}^{i n c} & =\int_{S} j \omega \mu_{1} \vec{J} G_{1} d S-\frac{j}{\omega \varepsilon_{1}} \int_{S} \nabla_{S}^{\prime} \cdot \vec{J} \nabla^{\prime} G_{1} d S+\int_{S} \vec{M} \times \nabla^{\prime} G_{1} d S \\
0 & =\int_{S} j \omega \mu_{2} \vec{J} G_{2} d S-\frac{j}{\omega \varepsilon_{2}} \int_{S} \nabla_{S}^{\prime} \cdot \vec{J} \nabla^{\prime} G_{2} d S+\int_{S} \vec{M} \times \nabla^{\prime} G_{2} d S
\end{aligned}
$$

\section{BIOELECTROMAGNETICS APPLICATION}

Some computational examples obtained using SIE formulation (36) related to the assessment of the induced field in the human brain are presented in this section.

The first example is related to the induced electric field in the brain of adult, 10-year old, and 5-year old child, respectively, exposed to horizontally polarized incident plane wave of $900 \mathrm{MHz}$, as shown in Fig. 3 [12].

It can be seen that at horizontally polarized incident wave, the higher values of induced electric field are obtained in smaller brain models in the frontal lobe areas.

The second example gives the comparison of the induced electric field in the same models, due to a transcranial magnetic stimulation (TMS) coil positioned $1 \mathrm{~cm}$ over primary motor cortex [13], as shown on Fig. 4.

Although the rapid decrease in the induced electric field is evident in all brain models, Fig. 4 shows that for smaller ones, the place where electric field falls to half its maximum value is moved closer to the surface. 

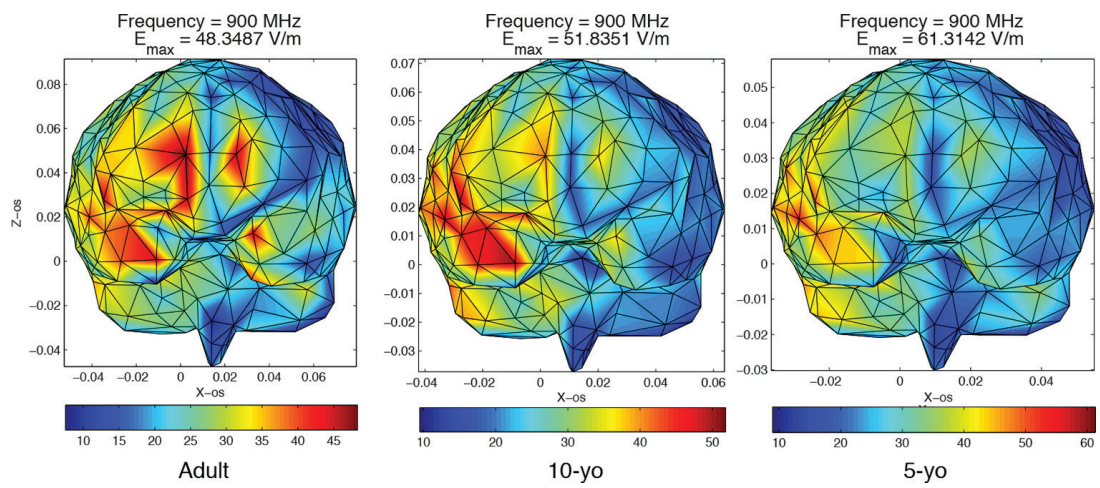

Figure 3: Comparison of the induced electric field due to plane EM wave.

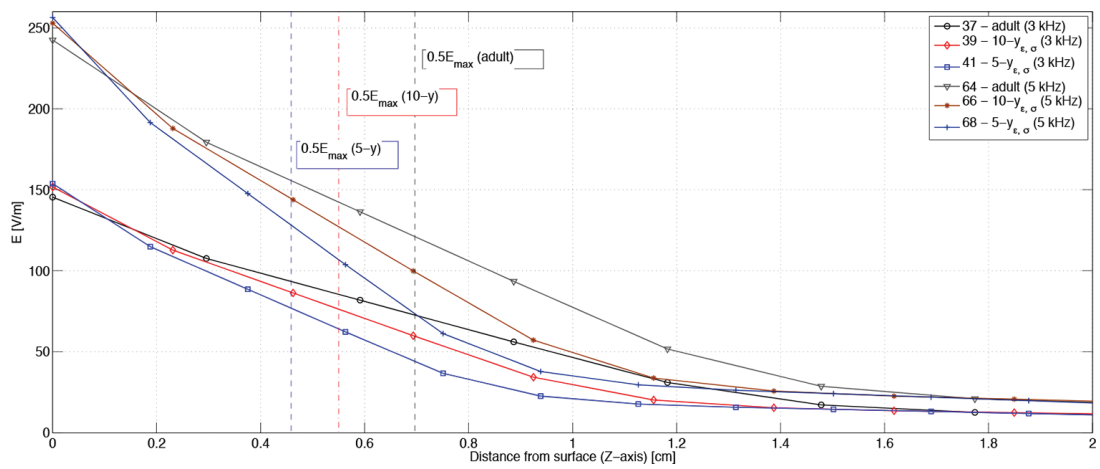

Figure 4: Comparison of the induced electric field due to TMS coil.

\section{CONCLUSION}

The paper revisits the application of surface equivalence theorem in the derivation of the EFIE formulation. The resulting coupled SIE set, although known for some problems arising in the CEM community, has only recently begun to appear in the bioelectromagnetics, offering a powerful, accurate and efficient alternative to the de facto standardly used differential equation based methods. Some computational examples obtained using the SIE based method are presented in the paper. The proposed method could be found useful in the initial assessment of the human brain exposed to EM radiation, while more realistic scenarios would require the use of a non-homogeneous models, resulting in the appropriate volume integral equation based formulation and the application of the volume equivalence principle.

\section{REFERENCES}

[1] Hand, J., Modelling the interaction of electromagnetic fields (10 MHz- $10 \mathrm{GHz})$ with the human body: methods and applications. Physics in Medicine and Biology, 53(16), pp. R243-R286, 2008.

https://doi.org/10.1088/0031-9155/53/16/r01 
[2] Poljak, D., Cavka, D., Dodig, H., Peratta, C. \& Peratta, A., On the use of the boundary element analysis in bioelectromagnetics. Engineering Analysis with Boundary Elements, 49, pp. 2-14, 2014. https://doi.org/10.1016/j.enganabound.2014.02.008

[3] Poljak, D., Human Exposure to Electromagnetic Fields. WIT Press, Boston, 2003.

[4] Cvetković, M. \& Poljak, D., An efficient integral equation based dosimetry model of the human brain. Proceedings of EMC EUROPE 2014, Gothenburg, Sweden, 1-4 September 2014, pp. 375-380, 2014.

[5] Poljak, D., Cvetković, M., Peratta, A., Peratta, C., Dodig, H. \& Hirata, A., On some integral approaches in EM dosimetry. BIOEM 2016, 2016.

[6] Poggio, A.J. \& Miller, E.K., Integral equation solutions of three-dimensional scattering problems. Computer Techniques for Electromagnetics, 2.E., ed. R. Mittra, pp. 159264, 1987.

[7] Chew, W.C., Tong, M.S. \& Hu, B., Integral Equation Methods for Electromagnetic and Elastic Waves. Morgan \& Claypol Publishers, San Rafael, CA, 2009.

[8] Cvetković, M., Poljak, D. \& Haueisen, J., Analysis of transcranial magnetic stimulation based on the SIE formulation. IEEE Transactions on Biomedical Engineering, 62(6), pp. 1535-1545, 2015.

https://doi.org/10.1109/tbme.2015.2393557

[9] Wei, F. \& Yilmaz, A., A more scalable and Efficient parallelization of the adaptive integral method - Part II: BIOEM Application. IEEE Transactions on Antennas and Propagation, 62(2), pp. 727-738, 2014.

https://doi.org/10.1109/tap.2013.2291564

[10] Stratton, J.A. \& Chu, L.J., Diffraction theory of electromagnetic waves. Physical Review, 56(1), pp. 99-107, 1939.

https://doi.org/10.1103/physrev.56.99

[11] Chen, K.M., A mathematical formulation of the equivalence principle. IEEE Transactions on Microwave Theory and Techniques, 37(10), pp. 1576-1581, 1989. https://doi.org/10.1109/22.41004

[12] Cvetković, M. \& Poljak, D., Electromagneticthermal dosimetry comparison of the homogeneous adult and child brain models based on the SIE approach. Journal of Electromagnetic Waves and Applications, 29(17), pp. 2365-2379, 2015. https://doi.org/10.1080/09205071.2015.1092896

[13] Cvetković, M., Poljak, D., Rogić Vidaković, M. \& Đogaš, Z., TMS induced fields in different brain models. Journal of Electromagnetic Waves and Applications, 30(14), pp. 1820-1835, 2016.

https://doi.org/10.1080/09205071.2016.1216807 